\title{
WAKE FIELD CALCULATION FOR THE TTF-FEL BUNCH COMPRESSOR SECTION
}

\author{
K. Rothemund, U. van Rienen \\ Institut für Allgemeine Elektrotechnik, Universität Rostock \\ Albert Einstein-Straße 2, D-18051 Rostock, Germany
}

\section{Abstract}

The TTF Free Electron Laser needs very short bunches to produce self-amplified spontaneous emission (SASE). This short bunch length is produced in a magnetic bunch compressor where the trajectories of particles with different energy have different path length in a way that the bunch is longitudinally compressed. As a parasitic effect the wake fields produced by the passing bunch will have the possibility to interact with the bunch itself and cause emittance growth. The high frequency behaviour of the beam pipe in the bunch compressor has to be analysed in order to identify trapped higher order modes (HOM) and to estimate beam distortion. Because of the complexity of the bunch compressor section direct eigenmode calculation is not possible due to lack of available computer power. A technique is presented which allows to compute eigenmodes of rf-structures by using scattering-parameters of subsections of the bunch compressor. This is done numerically based on the computer code MAFIA to model the different sections of the beam pipe.

\section{INTRODUCTION}

Numerical eigenmode calculations for complex components, such as the TTF bunch compressor, are limited by the available computer power, especially the available storage. In this paper a hybrid technique is presented to calculate eigenmodes and wakefields of geometrically complex structures via domain decomposition and mode matching.

The bunch compressor splits up into several subsections which are less complex and may be simulated individually with much smaller numerical effort of each single run. Since only the complete closed structure results in the eigenvalue problem determining eigenmodes and -frequencies, a splitting has to be done in terms of waves propagating between the sections described here using scattering-(S-)parameters.

\section{BASIC IDEA}

A rf-component can be represented by scattering- or Sparameters which describe signal reflection and transmission between each of the ports. It can be summerized in the scattering-matrix $\mathbf{S}$ :

$$
\vec{b}=\left(\begin{array}{ccc}
S_{11} & \cdots & S_{1 n} \\
\vdots & & \vdots \\
S_{n 1} & \cdots & S_{n n}
\end{array}\right) \vec{a}
$$

with $\vec{a}=\left(a_{1}, \ldots, a_{n}\right)^{T}$ representing all input- and $\vec{b}=$ $\left(b_{1}, \ldots, b_{n}\right)^{T}$ the output signals. $S_{i j}$ describes the transmission of the signal from port $i$ to $j$; it is a complex function of frequency: $S_{i j}=\left|S_{i j}(\omega)\right| \exp \left\{j \varphi_{i j}(\omega)\right\}$

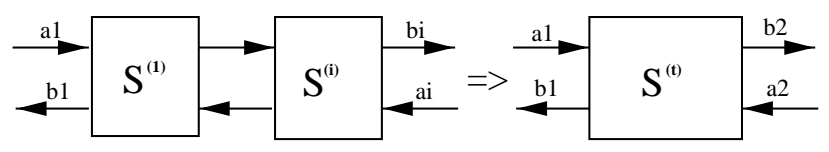

Figure 1: Describing a chain of rf-components with Sparameters.

The S-parameter formalism allows to connect several structures by combining their S-parameters (see [1]):

$$
\mathbf{S}^{(\mathbf{t})}=\mathbf{S}^{(\mathbf{1})} \odot \mathbf{S}^{(2)} \odot \mathbf{S}^{(3)} \ldots
$$

To solve for a resonator the open ports of the structure have to be closed which yields an eigenvalue problem. This is done by setting $a_{1}=r_{1} b_{1}$ and $a_{2}=r_{2} b_{2}$ and leads to

$$
\left(\begin{array}{l}
b_{1} \\
b_{2}
\end{array}\right)=\left(\begin{array}{cc}
S_{11}^{(t)} & S_{12}^{(t)} \\
S_{21}^{(t)} & S_{22}^{(t)}
\end{array}\right)\left(\begin{array}{cc}
r_{1} & 0 \\
0 & r_{2}
\end{array}\right)\left(\begin{array}{l}
b_{1} \\
b_{2}
\end{array}\right)
$$

For example setting $r_{1}=r_{2}=-1$ will create a loss free electric short cut at both ports. In general $r_{1}$ and $r_{2}$ also depend on $\omega$.

The eigenfrequencies are given by the solutions of

$$
\operatorname{det}(\mathbf{S R}-\mathbf{E})=0
$$

with $R=\left(\begin{array}{cc}r_{1} & 0 \\ 0 & r_{2}\end{array}\right)$ and $\mathbf{E}$ the unity matrix. This is equivalent to

$$
\begin{array}{r}
S_{11}^{(t)} r_{1} S_{22}^{(t)} r_{2}-S_{11}^{(t)} r_{1}-S_{22}^{(t)} r_{2}+ \\
1-S_{21}^{(t)} r_{1} S_{12}^{(t)} r_{2}=0
\end{array}
$$

\section{SIMPLE TEST EXAMPLE}

To verify the formulation given above a handy test geometry was modelled (see Fig. 2). Its eigenmodes could be 


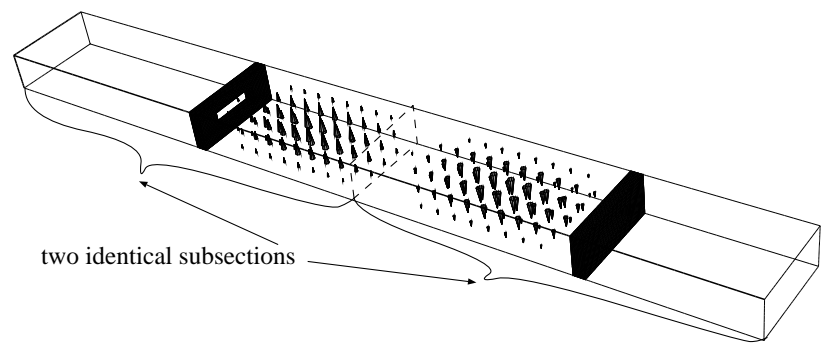

Figure 2: Test geometry with the $\vec{E}$-field of the third mode found by MAFIA, the boundaries are closed electrically.

calculated directly with the MAFIA [2] E-module. Both ports are closed electrically $\left(r_{1}=r_{2}=-1\right)$. The test geometry consists of two identical subsections of waveguide, each with an asymmetrically placed iris.

Assuming a reciprocal $\left(S_{m n}=S_{n m}\right)$ and loss-free $\left(\mathbf{S}^{*} \mathbf{S}=\mathbf{E}\right)$ structure in the simple case of a single propagating mode equation (5) leads to the following equation for the eigenfrequencies $\omega_{0}$ :

$$
\sin \left(\varphi_{11}^{(t)}\left(\omega_{0}\right)-\varphi_{12}^{(t)}\left(\omega_{0}\right)\right)=\left|S_{11}^{(t)}\left(\omega_{0}\right)\right| \sin \left(\varphi_{12}^{(t)}\left(\omega_{0}\right)\right)
$$

The S-parameters of one subsection were calculated with MAFIA in time domain for frequencies of $1.2 \ldots 1.6 \mathrm{GHz}$.

Fig. 3 shows the plot of the left and right hand side of (6) in this interval. The eigenfrequencies are given by the intersections of both plots. As shown in table 1 the direct eigenmode calculation and the calculation via S-parameters correspond very good.

The presented procedure allows to divide a complex structure into smaller subsections for which S-parameters can be calculated with a reasonable amount of computer power. Combining the single results yields the S-parameter matrix of the total structure and their eigenfrequencies.

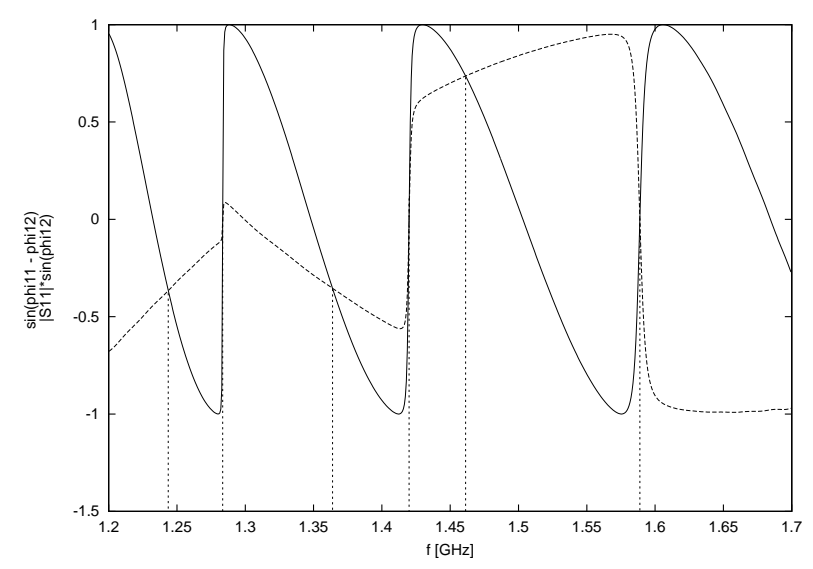

Figure 3: Plot of $\sin \left(\varphi_{11}^{(t)}-\varphi_{12}^{(t)}\right)=\left|S_{11}^{(t)}\right| \sin \left(\varphi_{12}^{(t)}\right)$ versus the frequency $(f=1.2 \ldots 1.6 \mathrm{GHz})$.

\begin{tabular}{l|l|c} 
S-parameters & MAFIA E-mod. & rel. error in \% \\
\hline $1.243639 \mathrm{GHz}$ & $1.243016 \mathrm{GHz}$ & 0.50 \\
$1.283487 \mathrm{GHz}$ & $1.283967 \mathrm{GHz}$ & -0.04 \\
$1.363857 \mathrm{GHz}$ & $1.363793 \mathrm{GHz}$ & 0.005 \\
$1.419875 \mathrm{GHz}$ & $1.420691 \mathrm{GHz}$ & -0.06 \\
$1.461280 \mathrm{GHz}$ & $1.459711 \mathrm{GHz}$ & 0.11 \\
$1.588825 \mathrm{GHz}$ & $1.589970 \mathrm{GHz}$ & -0.07
\end{tabular}

Table 1: Comparison of the eigenfrequencies found by the S-parameter method and by MAFIA E-module.

\section{THE TTF-FEL BUNCH COMPRESSOR}

Figure 4 shows the bunch compressor section with the two junctions and the middle chamber. The straight part of the beam pipe is not modelled because its cut-off frequency $f_{c o}$ is $3.0 \mathrm{GHz}$. So there are no propagating modes in the frequency range of $1.6 \ldots 2.2 \mathrm{GHz}$. The middle chamber itself can be subdivided again: two steps, two bellows and a flange used for a vacuum pump and a beam position monitor.

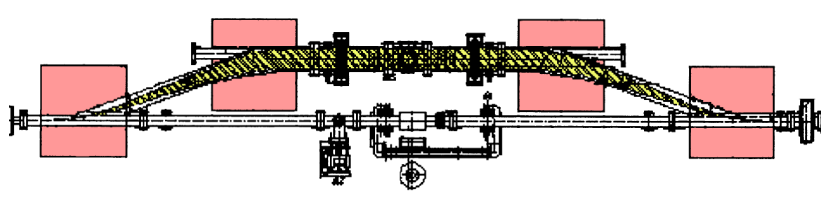

Figure 4: The TTF-FEL bunch compressor section.

\subsection{Exploiting the Symmetry}

The overall length of the bunch compressor is about 4.5 meters, whereas some of the subsections contain structures with a dimension in the order of $5 \mathrm{~mm}$ (bellows). This may illustrate the difficulty of proper discretisation of the complete structure.

The subsections can be chosen in such a way that they show symmetries (except the step). This allows to calculate the full S-parameter matrix in a single run.

\subsection{Terminating the Structure}

The beam pipes at the outermost parts of the junctions are rectangular waveguides with a cut-off frequency of 3.0 GHz. The cut-off frequency of the inner part of the junction section is $1.66 \mathrm{GHz}$, cut-off frequency of the second propagating mode in the middle section is $2.32 \mathrm{GHz}$. Therefore a frequency range of $1.7 \ldots 2.2 \mathrm{GHz}$ was chosen for this calculation. Within this frequency range multimodal wave propagation can be excluded and the junctions "naturally" terminate the structure. Thus they are described by a single quantity, a frequency dependent reflection coeffient $r(\omega)$. 
With $r_{1}=r_{2}=r$ the equation for the eigenfrequencies then turns into

$$
\begin{array}{r}
\left(S_{11}^{(t)}\left(\omega_{0}\right) S_{22}^{(t)}\left(\omega_{0}\right)-S_{12}^{(t)}\left(\omega_{0}\right) S_{21}^{(t)}\left(\omega_{0}\right)\right) r^{2}\left(\omega_{0}\right)- \\
\left(S_{11}^{(t)}\left(\omega_{0}\right)+S_{22}^{(t)}\left(\omega_{0}\right)\right) r\left(\omega_{0}\right)+1=0
\end{array}
$$

\subsection{Results}

All S-parameters were calculated using MAFIA's time domain solver T3. Due to different mesh and time steps the data set of each S-parameter calculation has different frequency steps. This requires interpolation when concatenating the subsections. (cf. eq. (2)):

The eigenfrequencies found by examinating the resulting data set (linear interpolation) are 1.79449, 1.81490, $1.82200,1.84152,1.86134,1.88619,1.90186,1.91547$, $1.95155,1.95658,2.02550,2.07312,2.08820,2.10578$, $2.11272,2.13198$ and $2.16954 \mathrm{GHz}$. Fig.5 displays a plot of the real and imaginary part of the left hand side of (7) in the frequency range of $2.0 \ldots 2.1 \mathrm{GHz}$ (upper plot) with eigenfrequencies at 2.02550, 2.07312 and $2.08820 \mathrm{GHz}$. The lower plot (frequency: $2.0 \ldots 2.1 \mathrm{GHz}$ ) shows eigenfrequencies at 2.10578, 2.11272, 2.13198 and $2.16954 \mathrm{GHz}$.
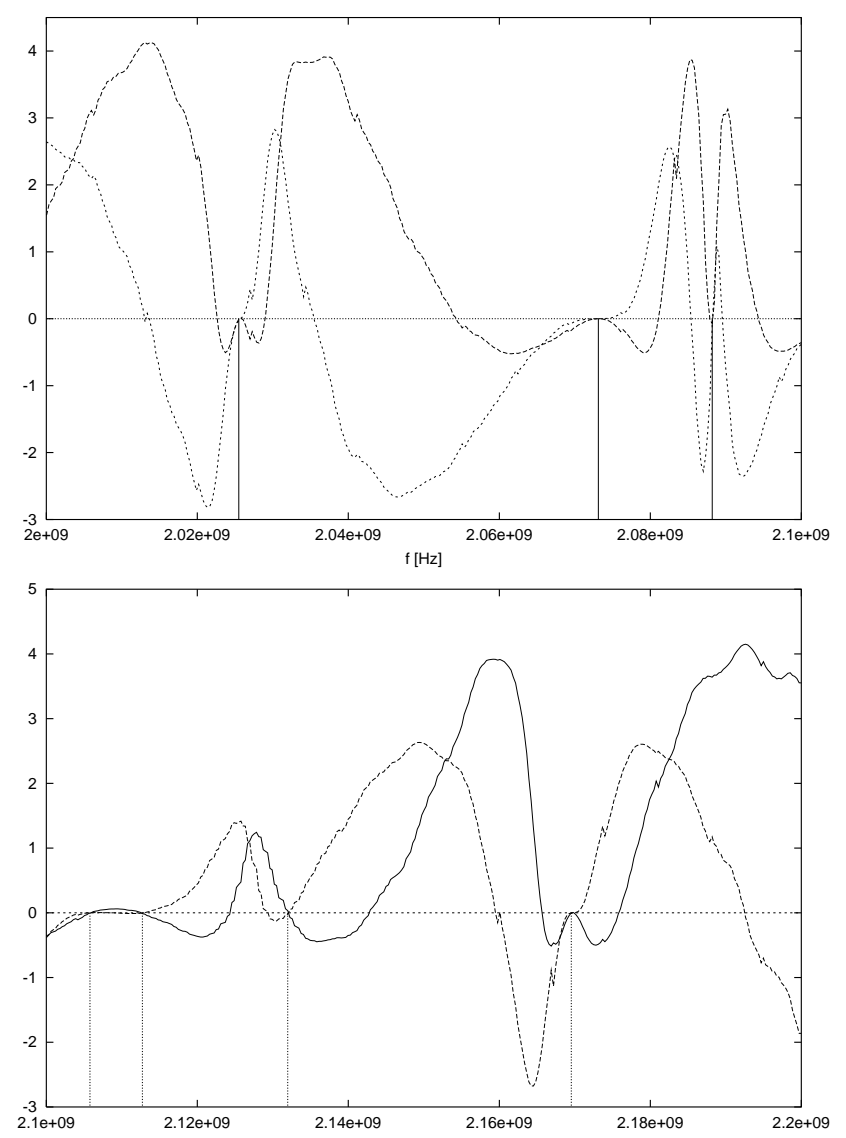

Figure 5: Real and imaginary part of the left hand side of (7) vs. frequency.

\section{CONCLUSION}

The S-parameter formalism offers a possibility to calculate eigenfrequencies of a structure that is too complex for direct simulation. A validation for this procedure was given in the case of a simple test geometry. Even though the overall effort of this method is higher than that of the direct calculation this procedure enables the computation of eigenfrequencies of complex structures in cases where this would exceed the limits given by computer storage usually available.

As described above, for the time being, the frequencies calculated for the bunch compressor section can not be compared with direct eigenmode calculation but have to be measured for validation.

\section{PROSPECT}

To calculate the wakefields using the mode matching method furthermore the electromagnetic fields corresponding to the eigenfrequencies have to be known. They can be determined by exciting monochromatic waves with the eigenfrequencies at each port of each section and calculating the fields in the steady state. Superposition of the steady state fields yields the eigenmode fields of the total structure in each component.

\section{ACKNOWLEDGEMENT}

The author would like to thank H.-W. Glock for many fruitful discussions.

\section{SUPPORT}

This work was supported by DESY, Hamburg.

\section{REFERENCES}

[1] U. van Rienen, Higher Order Mode Analysis of Tapered Disc-Loaded Waveguides using the Mode Matching Technique, Particle Accelerators, 1993

[2] MAFIA V4.0, CST GmbH, Lautenschlägerstr. 38, D-64289 Darmstadt 